\title{
Analysis of Subtopic Discovery Algorithms for Real-time Information Summarization
}

\author{
Gustavo Gonçalves \\ NOVA LINCS, Dep. Computer Science \\ Universidade NOVA de Lisboa \\ gs.goncalves@campus.fct.unl.pt
}

\author{
Flávio Martins \\ NOVA LINCS, Dep. Computer Science \\ Universidade NOVA de Lisboa \\ flaviomartins@acm.org
}

\author{
João Magalhães \\ NOVA LINCS, Dep. Computer Science \\ Universidade NOVA de Lisboa \\ jm.magalhaes@fct.unl.pt
}

\begin{abstract}
The rise of large data streams introduces new challenges regarding the delivery of relevant content towards an information need. This need can be seen as a broad topic of information. By identifying sub-streams within a broader data stream, we can retrieve relevant content that matches the multiple facets of the topic; thus summarizing information, and matching the initial need.

In this paper, we propose to study the generation of sub-streams over time and compare various aggregation methods to summarize information. Our experiments were made using the standard TREC Real-Time Summarization (RTS) 2017 dataset.

\section{ACM Reference Format:}

Gustavo Gonçalves, Flávio Martins, and João Magalhães. 2018. Analysis of Subtopic Discovery Algorithms for Real-time Information Summarization. In WWW'18 Companion: The 2018 Web Conference Companion, April 23-27, 2018, Lyon, France. ACM, New York, NY, USA, 2 pages. https://doi.org/10. $1145 / 3184558.3191651$
\end{abstract}

\section{INTRODUCTION}

The continuous generation of content in social-media networks originates real-time data streams that need to be filtered and analyzed, to identify relevant information towards end users. The TREC RTS track aims to tackle these issues through the delivery of the most relevant tweets in a near real-time push notifications scenario, or in a delayed digest in the daily summarization scenario. The two scenarios share the criteria of delivering relevant and diverse information. This track introduces new challenges such as, dealing with a dataset that is continuously expanding and prepare algorithms to face different relevance distributions over time.

The top two ranking teams $[3,4]$ of the 2016 edition of the track, leveraged on a threshold definition for the detection of diverse documents. Both teams defined a similarity measure and trained their model on the TREC Microblog 2015 dataset, to set a diversity threshold. For the 2017 edition of the RTS track, we followed a similar approach for summarizing topics.

The real-time constraints of the track will dictate the strategy to detect diverse documents. The two main strategies consist in the comparison between all submitted tweets, or between a tweet

This work has been partially funded by the CMU Portugal research project Ref. GoLocal CMUP-ERI/TIC/0033/2014, the project NOVA LINCS Ref. UID/CEC/04516/2013 and FCT-MEC of Portugal.

This paper is published under the Creative Commons Attribution 4.0 International (CC BY 4.0) license. Authors reserve their rights to disseminate the work on their personal and corporate Web sites with the appropriate attribution.

WWW' 18 Companion, April 23-27, 2018, Lyon, France

() 2018 IW3C2 (International World Wide Web Conference Committee), published under Creative Commons CC BY 4.0 License.

ACM ISBN 978-1-4503-5640-4/18/04

https://doi.org/10.1145/3184558.3191651 and the vocabulary set of the summary. Despite the document-todocument comparison improved performance when compared with document-to-set approach [1], the latter is less resource expensive which can be crucial in a mobile scenario.

With our participation, we studied the impact of different summarization approaches. The summarization study was further explored through the utilization of clustering and topic models. We aimed to assess the effectiveness of standard clustering and topic modeling methods when faced with a text stream dataset. The summarization techniques are described in Section 2, the achieved results are presented in Section 3, and the conclusions in Section 4.

\section{DISCOVERING SUBTOPICS}

Diversity is measured by the similarity between documents and is one of the principal criteria to evaluate the performance of the systems participating in the TREC RTS track. The relevance assessment of the RTS track relies on semantic clustering. The semantic clustering [2] was made by the National Institute of Standards and Technology (NIST) assessors, which focused on grouping tweets that present similar content. The relevance evaluation using clustering penalizes the submission of similar tweets that appear after the submission of a seminal tweet. In this paper, we use the notation of cluster interchangeably with subtopic. To achieve a perfect score, systems must submit the most relevant tweet of each assessed cluster. We have chosen to study the following popular algorithms, K-means, NMF, LDA, and DTM.

\section{EVALUATION}

To evaluate our work we needed to clearly define the dataset and protocol that were considered. The chosen metrics to measure the clustering effectiveness were NMI and ARI, and for the relevance of the summarization results we leveraged on nDCG@10.

\subsection{Dataset and Protocol}

The utilized dataset was built by listening to the Twitter $1 \%$ stream during eight days, between 29th of July and 5th of August of 2017, UTC. This dataset was utilized in the TREC RTS track. We leveraged on the relevance judgments made by the NIST assessors, both regarding the tweets relevance and the cluster assignments.

We defined our task as the comparison of the grouping performance, in a fine-grained scenario, of commonly utilized clustering and topic modeling techniques. In our experiments, we wanted to observe how close the standard techniques would approximate the ideal clustering for the RTS track.

We produce a common retrieval ranking for all methods by filtering out non-relevant documents according to the relevance judgments. We simulated the perfect diversification algorithm to 

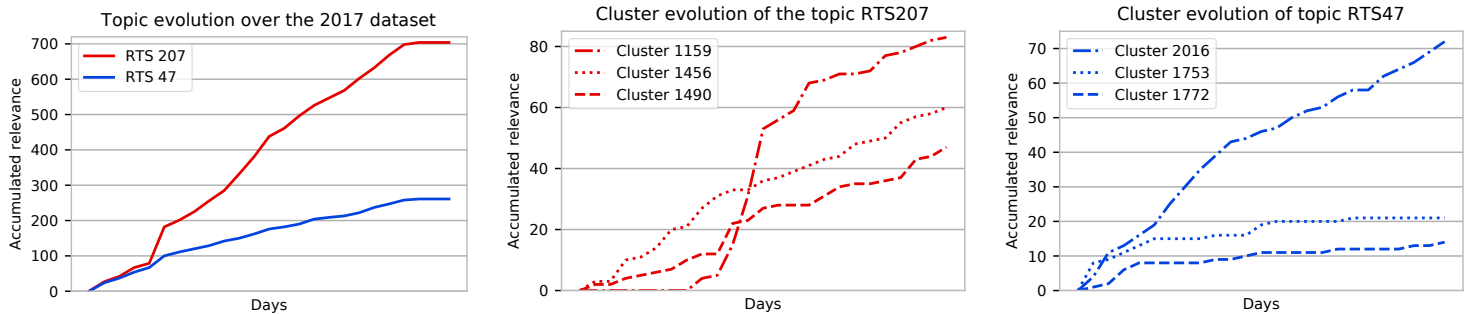

Figure 1: Drilldown relevance study of the chosen subtopics contained in topics RTS207 (red) and RTS47 (blue).

compare the algorithms. For each identified subtopic, the document with the highest score would be submitted to evaluate relevance.

\subsection{Results and Discussion}

We focused our analysis in two main components. The subtopic evolution through time, and the algorithms' performance to discover subtopics.

3.2.1 Subtopic evolution. In Figure 1 we illustrate the evolution of the accumulated relevance of topics, RTS207 and RTS47, over the evaluation period. The first plot presented on the left depicts the total accumulated relevance of the topics. The following plots present the relevance evolution of the three clusters, with a higher accumulated relevance for the chosen topics. Each document could be evaluated as not relevant, relevant, and highly-relevant.

We can observe three relevance distributions, by analyzing the three subtopics of each topic, that algorithms must be able to detect. First, we can observe subtopics with a reasonably uniform growth rate, e.g., subtopics 1456 and 1490 of topic RTS207, and subtopic 2016 of topic RTS47. This behavior can be interpreted as sub-streams of interest that are being updated during the evaluation period.

Secondly, we can see subtopics that have a spike in accumulated relevance in a short time span, e.g., subtopic 1159 of topic RTS47. This subtopic distribution can be explained as events that became main sub-streams of the topic being studied, due to the intense publication of documents in social-media.

Finally, we observe that some subtopics reach a plateau earlier, e.g., subtopics 1753 and 1772 of topic RTS47. These can be interpreted as sub-streams of interest that had an impact towards the main topic, yet were isolated events without follow-up, or fading events before the beginning of the evaluation period.

Retrieval systems are typically directed to find all or the most relevant documents. However, the delivery of real-time information presents more subtle challenges, which depend on the prediction of incoming information. Hence, new clustering and diversity approaches must be studied to meet the requirements introduced by these real-time relevance distributions.

3.2.2 Subtopic discovery. For the discovery of subtopics, we leveraged on the NMI and ARI metrics to assess the similarity between the obtained and ideal clusters. For each algorithm, we provided the optimal number of subtopics, for each topic.

In Table 1 we can observe a comparison among the considered algorithms considering the chosen metrics. NMF is the method that approximated more closely the optimal clustering, surpassing
Table 1: Results of the impact of the clustering methods on the real-time summarization performance.

\begin{tabular}{lrrr}
\hline Method & NMI & ARI & nDCG@10 \\
\hline No clustering & - & - & 0.4681 \\
\hline K-Means JS(K=\#of subtopics) & 0.726 & 0.387 & 0.5771 \\
K-Means Cos(K=\#of subtopics) & 0.736 & 0.406 & 0.5771 \\
NMF & 0.740 & 0.421 & 0.5767 \\
LDA & 0.613 & 0.228 & 0.4426 \\
DTM & 0.658 & 0.267 & 0.4688 \\
\hline
\end{tabular}

all other metrics both in NMI and ARI. Followed by the K-Means variations, which performed closely to NMF, both in NMI and ARI, especially using the JS divergence. LDA and DTM performance was poor due to the lack of textual elements, and documents to train the model.

We found that NMF and K-Means worked better in the subtopic identification task for short documents. Nevertheless, there is still room for improvement, with both methods diverging from the perfect clustering.

\section{CONCLUSION}

The analysis of different data grouping methods allowed us to conclude which of the currently used methods are more suitable towards the discovery of sub-streams of interest. The shortness of the documents presents itself as a challenge towards this objective. NMF presented good performance in the simulated post hoc task, using the oracle condition of knowing the number of subtopics in advance. Further work is needed to determine a suitable similarity metric, sensitive to small yet meaningful changes in document vocabulary. With a more accurate similarity, an improved document summarization can be achieved. Ultimately, swiftly adapting to the subtle dynamics of the stream.

\section{REFERENCES}

[1] Margarita Karkali, François Rousseau, Alexandros Ntoulas, and Michalis Vazirgiannis. 2014. Using Temporal IDF for Efficient Novelty Detection in Text Streams. CoRR abs/1401.1456 (2014)

[2] Jimmy J. Lin, Salman Mohammed, Royal Sequiera, Luchen Tan, Nimesh Ghelani, Mustafa Abualsaud, Richard McCreadie, Dmitrijs Milajevs, and Ellen M. Voorhees. 2017. Overview of the TREC 2017 Real-Time Summarization Track. In TREC 2017. National Institute of Standards and Technology.

[3] Reem Suwaileh, Maram Hasanain, and Tamer Elsayed. 2016. Light-Weight, Conservative, yet Effective: Scalable Real-Time Tweet Summarization. In TREC 2016. National Institute of Standards and Technology.

[4] Haihui Tan, Dajun Luo, and Wenjie Li. 2016. PolyU at TREC 2016 Real-Time Summarization. In TREC 2016. National Institute of Standards and Technology. 